Revista Brasil. Bot., V.33, n.1, p.21-36, jan.-mar. 2010

\title{
Floristic and vegetation structure of a granitic grassland in Southern Brazil
}

\author{
PEDRO MARIA DE ABREU FERREIRA', SANDRA CRISTINA MÜLLER ${ }^{2}$, \\ ILSI IOB BOLDRINI ${ }^{3}$ and LILIAN EGGERS 3,4
}

(received: August 07, 2008; accepted: October 28, 2009)

\begin{abstract}
Floristic and vegetation structure of a granitic grassland in Southern Brazil). A floristic and structural survey of a natural grassland community was conducted on Morro do Osso, a granitic hill in Porto Alegre, RS, Brazil. Structural data were surveyed in 39 one square meter plots placed over two major grassland areas. An accidental fire has occurred in one of the areas approximately one year prior to our survey, leading to further analysis of parameters differences between sites. The floristic list contains 282 species, whereas the structural survey has found 161 species. Families with highest accumulated importance values were Poaceae, Asteraceae and Fabaceae. The diversity and evenness indexes were 4.51 nats ind $^{-1}$ and 0.86 , respectively. Cluster analysis denoted two groups coinciding with the areas distinguished by the fire disturbance. A similarity analysis between our data and two other data sets from nearby granitic hills resulted in $28 \%$ to $35 \%$ similarity, with equivalent species-family distribution and many common dominant species, corroborating the concept of a continuous flora along the South Brazilian granitic hills.
\end{abstract}

Key words - campos, diversity, fire, relict vegetation, similarity, threatened species

RESUMO - (Florística e estrutura da vegetação de um campo granítico no Sul do Brasil). Um levantamento florístico e estrutural de uma comunidade de campo natural foi conduzido no Morro do Osso, um morro granítico em Porto Alegre, RS, Brasil. Os dados estruturais foram obtidos em 39 quadros de $1 \mathrm{~m}^{2}$, dispostos em duas grandes áreas de campo. Uma queimada acidental ocorreu em uma das áreas, aproximadamente um ano antes do levantamento, propiciando a análise de diferenças entre os parâmetros dos dois sítios de amostragem. A lista florística contém 282 espécies e, no levantamento estrutural, foram encontradas 161 espécies. As famílias com maior valor de importância acumulado foram Poaceae, Asteraceae e Fabaceae. Os índices de diversidade e a equabilidade foram 4,51 nats ind ${ }^{-1} \mathrm{e} 0,86$, respectivamente. A análise de agrupamento identificou dois grupos nítidos, que coincidem com os locais atingidos ou não pelo fogo. Uma análise de similaridade entre os dados obtidos e dois outros conjuntos de dados de morros graníticos vizinhos resultou em $28 \%$ a $35 \%$ de similaridade, com uma equivalente distribuição de espécies por família e muitas espécies dominantes comuns, corroborando a noção de continuidade da flora ao longo dos morros graníticos no sul do Brasil.

Palavras-chave - campos, diversidade, espécies ameaçadas, fogo, similaridade, vegetação relictual

\section{Introduction}

The study of natural grassland communities, despite its well known ecological importance, has been historically neglected (Overbeck et al. 2007) or limited to dominant grass species used in livestock production (Uys et al. 2004) or grain monocultures. As it is, there is a lack of floristic and structural studies concerning these communities worldwide, which leads, at least partly,

1. Universidade Federal do Rio Grande do Sul - Programa de PósGraduação em Botânica, Av. Bento Gonçalves, 9500, Bloco IV, Prédio 43433, 91501-970 Porto Alegre, RS, Brazil.

2. Universidade Federal do Rio Grande do Sul, Instituto de Biociências, Departamento de Ecologia, Av. Bento Gonçalves, 9500, Caixa Postal 15007, 91501-970 Porto Alegre, RS, Brazil.

3. Universidade Federal do Rio Grande do Sul, Instituto de Biociências, Departamento de Botânica, Av. Bento Gonçalves, 9500, Bloco IV, Prédio 43433, 91501-970 Porto Alegre, RS, Brazil.

4. Corresponding author: lilian.eggers@ufrgs.br to an underestimation of their richness, diversity and conservational value.

Subtropical grasslands, which are also called campos in Brazil (Behling 2002, Focht \& Pillar 2003, Overbeck et al. 2007), were dominant in southern Brazilian landscape during late Pleistocene, and its present distribution can be interpreted as a remnant of a drier and cooler climate in the region (Behling 2002, Bredenkamp et al. 2002). Thus, campos configure a relict formation, and the knowledge of its floristic and structural features is vital to understand the processes that lead to modern landscape. Rambo (1954) emphasized both the richness and ecological importance of the southern Brazilian herbaceous flora by indicating that herbaceous species of Asteraceae alone are more numerous than the entire local woody flora.

Despite its ecological and social-economic importance, natural grasslands are a historic target of many anthropogenic disturbances, such as land 
conversion to grain and wood monocultures, overgrazing and unrestricted fire, being often wiped away from the landscape even before being well known by science. Nevertheless, fire disturbances not always have hazardous consequences for plant communities, such as depletion of soil nutrients and local plant extinction, and there is growing evidence of the importance of both natural and human-driven fires for the maintenance of many natural grassland communities (Quadros \& Pillar 2001, Uys et al. 2004, Overbeck et al. 2005, Overbeck et al. 2006, Behling et al. 2007, Müller et al. 2007). Moreover, the acknowledgement of fire disturbance as a crucial environmental factor to the very existence of subtropical grasslands is increasing (Quadros \& Pillar 2001, Behling 2002, Behling et al. 2004, Overbeck et al. 2005, Müller et al. 2007).

Granitic grassland formations in southern Brazil includes an area beside the city of Porto Alegre, Rio Grande do Sul, represented by a series of granitic hills known as Morro do Osso, Morro Santana, Morro da Polícia, Morro São Pedro, among others. Although recent floristic and structural surveys concerning southern Brazilian granitic grassland formations are scarce, a historical analysis of the Porto Alegre flora can be found in Rambo (1954), who displayed an extensive species list, albeit outdated nowadays. The most extensive approach was presented by Aguiar et al. (1986) concerning a preliminary floristic survey in ten granitic hills in Porto Alegre region. Boldrini et al. (1998) surveyed floristic and ecological aspects of a grassland community at Morro da Polícia and Overbeck et al. (2006) presented a local species list of Morro Santana, linking floristic composition of burned grassland to environmental factors, such as soil properties and distance from the forest border.

Working in a southern Brazilian granitic grassland, our objectives were: (1) to present floristic and structural data of the local herbaceous flora; (2) to explore the effects of an accidental fire event on floristic and structural patterns and (3) to compare plant diversity of similar hill's formation in the context of grassland vegetation dynamic.

\section{Material and methods}

Study area - The study was carried out on Parque Natural Morro do Osso ( $30^{\circ} 07^{\prime} \mathrm{S}, 51^{\circ} 14^{\prime} \mathrm{W}$; $143 \mathrm{~m}$ a.s.l.), an area inserted into a chain of granitic hills that surrounds the city of Porto Alegre, Rio Grande do Sul, Brazil. This geological formation, known by the presence of several rare and endemic plant species (Rambo 1954, Aguiar et al. 1986, Boldrini et al. 1998, Brack et al. 1998), configures an intersection point among four different structural provinces that shape the south Brazilian landscape: the Crystalline Shield, the Peripheral Depression, the bottom of the Seashore Plain and the Lagoon System (Menegat et al. 1998). Likewise, this granitic chain is historically thought to be responsible for notorious local plant endemism, acting like a refuge area during ocean transgression times (Rambo 1954). The study site is a Conservation Unit since 1994, covering 127 ha of natural area (Brack et al. 1998).

Natural vegetation at the site comprises a mosaic of grasslands and forests (Atlantic Rainforest), the first occurring mostly on hilltops and north or northwest slopes and the latter mostly along river courses and south or southeast slopes (Rambo 1954, 1956, Müller et al. 2007). Grasslands cover around $40 \%$ of the study area and fire, either natural or caused by neighboring human settlements, is a common disturbance to the ecosystem at spaced but non-fixed intervals. There are two major grassland patches in the study site, one is westoriented and the other is east-oriented, being separated by a relatively large forest/woodland area (figure 1). There is a record of significant burning on the east grassland area in 2005 , one year prior to the survey presented in this paper, confirmed by the first collected specimens at the site, which had clear signals of recent burning.
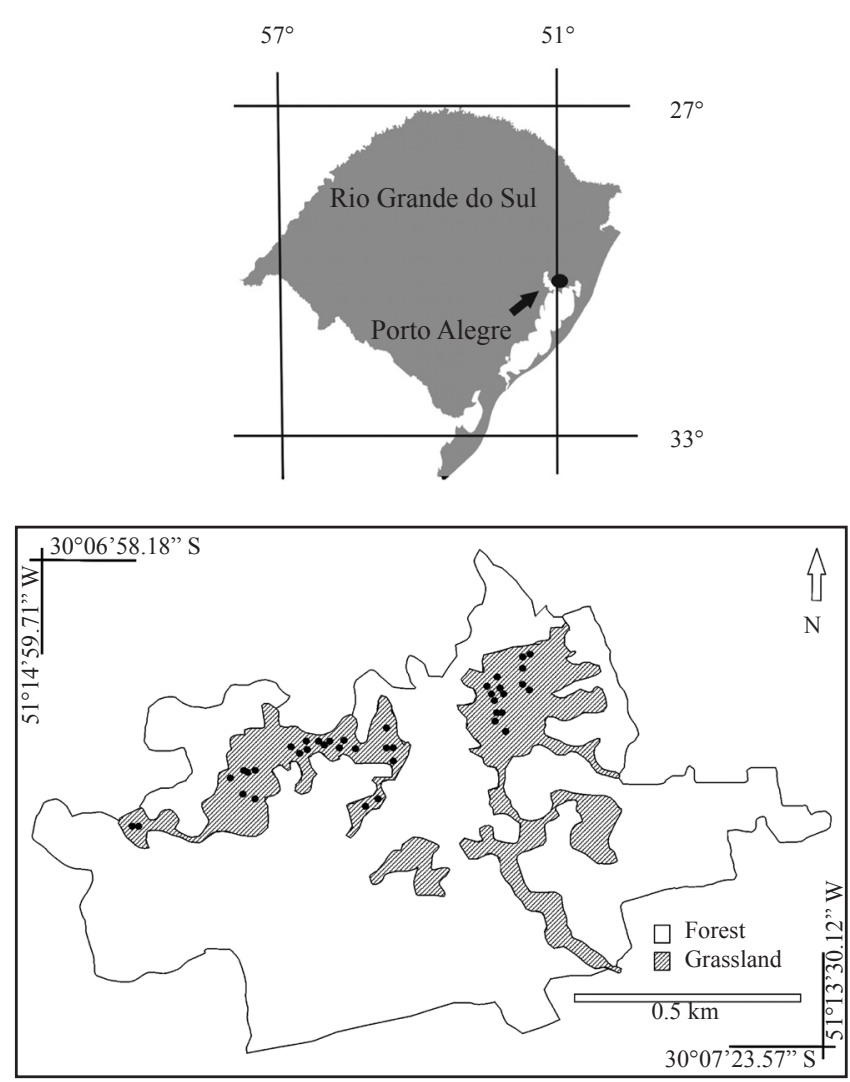

Figure 1. Spatial location of the study site Parque Natural Morro do Osso $\left(30^{\circ} 07^{\prime} \mathrm{S}, 51^{\circ} 14^{\prime} \mathrm{W}\right)$ and the Porto Alegre city, Rio Grande do Sul State, Brazil. Black dots on grassland areas represent (not in scale) the 39 sampling units. 
Climate at the study site is subtropical humid, with no dry season (Köppen's Cfa). The average annual temperature is $19.5^{\circ} \mathrm{C}$ and the mean annual precipitation is $1348 \mathrm{~mm}$ (Nimer 1990). The soil is granite-originated, composed by post-tectonic granitoids dated from 550 m.y.a. and known as Santana granite (Sestren-Bastos 2006).

Data collection and analysis - Data for the structural survey were collected in 39 one square meter plots randomly placed in two major grassland areas (figure 1). Other grassland-covered areas that can be seen in figure 1 comprise tracks, shrublands and surroundings of Park management's buildings, thus not included in the sampling area. In each plot, species cover was registered, applying the Daubenmire scale (Mueller-Dombois \& Ellenberg 1974). The survey took place in January 2006, although prior to that, other expeditions were made to search floristic records. Plant identification was done using specific bibliography and contacting specialists when necessary. Family circumscription follows APWeb (2003). A full species list for the Morro do Osso's grasslands is presented based on our own information and previous publications (Brack et al. 1998, Brack et al. 2001).

For each species, absolute and relative frequency (AF and $\mathrm{RF}$, respectively), absolute and relative cover (AC and $\mathrm{RC}$, respectively) and importance value (IV) were calculated. Shannon-Wiener diversity index (H') and the Pielou evenness (E) (Pielou 1969, Magurran 1988) were also obtained. Open soil and litter were evaluated in each plot unit, but omitted in the community indexes calculation.

The raw data matrix obtained for the grassland community (species plus open soil and litter per 39 sampling plots) was submitted to ordination and cluster analysis on Multiv software
(Pillar \& Orlóci 1993, 1996, Pillar 2006). Both methods were submitted to the bootstrap analysis to verify cluster groups sharpness and ordination axis stability (Pillar 1999a, b). The ordination method was the principal coordinate analysis (PCoA) and, in the cluster analysis, incremental sum of squares was used as the clustering criterion, both based on chord distance resemblance measure between plots (Podani 2000). Variables (species, litter and open soil cover) with axes correlation values greater than 0.45 were included in the ordination scatter diagram. The statistically supported groups obtained from this analysis were then submitted to separate phytosociological analysis with the previously mentioned parameters. Vegetation height, richness, open soil and litter were submitted to variance analysis comparing groups of plots by randomization test with 10,000 iterations (Pillar \& Orlóci 1996, Manly 1997).

The species list obtained in the structural survey was submitted to a similarity analysis (Jaccard similarity index) based on structural data from two neighboring granitic hills: Morro da Polícia (Boldrini et al. 1998) and Morro Santana (Overbeck et al. 2006). Only fully identified taxa (specific level) were included in this analysis. A nomenclatural revision was also done on the species lists in order to avoid synonyms and standardize data.

\section{Results}

Floristic and structural analysis - The floristic list contains 282 species, distributed in 42 families (table 1). Poaceae ( 74 species), Asteraceae (63 species) and Fabaceae (25 species) were the most representative families in the floristic survey.

Table 1. Floristic list of grassland formations at Morro do Osso. ( $\mathrm{F}=$ Species collected in field expeditions; $\mathrm{R}=\mathrm{Species}$ appointed for the study site by Brack et al. (1998; 2001); Ph = Species sampled in the structural survey).

\begin{tabular}{lll}
\hline Family & Species & \\
\hline Acanthaceae & Ruellia morongii Britton & $\mathrm{Ph}$ \\
& Stenandrium diphyllum Nees & $\mathrm{Ph}$ \\
Amaranthaceae & Gomphrena globosa L. & $\mathrm{R}$ \\
& Gomphrena graminea Moq. & $\mathrm{Ph}$ \\
& Pfafia tuberosa (Sprengel) Hicken & $\mathrm{Ph}$ \\
Amaryllidaceae & Habranthus gracilifolius Herb. & $\mathrm{F}$ \\
Anacardiaceae & Schinus weinmannifolius Engl. & $\mathrm{Ph}$ \\
Apiaceae & Apium leptophyllum F. Muell. ex Benth. & $\mathrm{F}$ \\
& Eryngium ciliatum Cham. \& Schltdl. & $\mathrm{Ph}$ \\
& Eryngium elegans Cham. \& Schltdl. & $\mathrm{F}$ \\
& Eryngium eriophorum Cham. \& Schltdl. & $\mathrm{R}$ \\
& Eryngium horridum Malme & $\mathrm{Ph}$ \\
& Eryngium pristis Cham. \& Schltdl. & $\mathrm{Ph}$ \\
& Eryngium sanguisorba Cham. \& Schltdl. & $\mathrm{F}$ \\
& Hydrocotyle umbellata L. & $\mathrm{R}$ \\
\end{tabular}


continuation

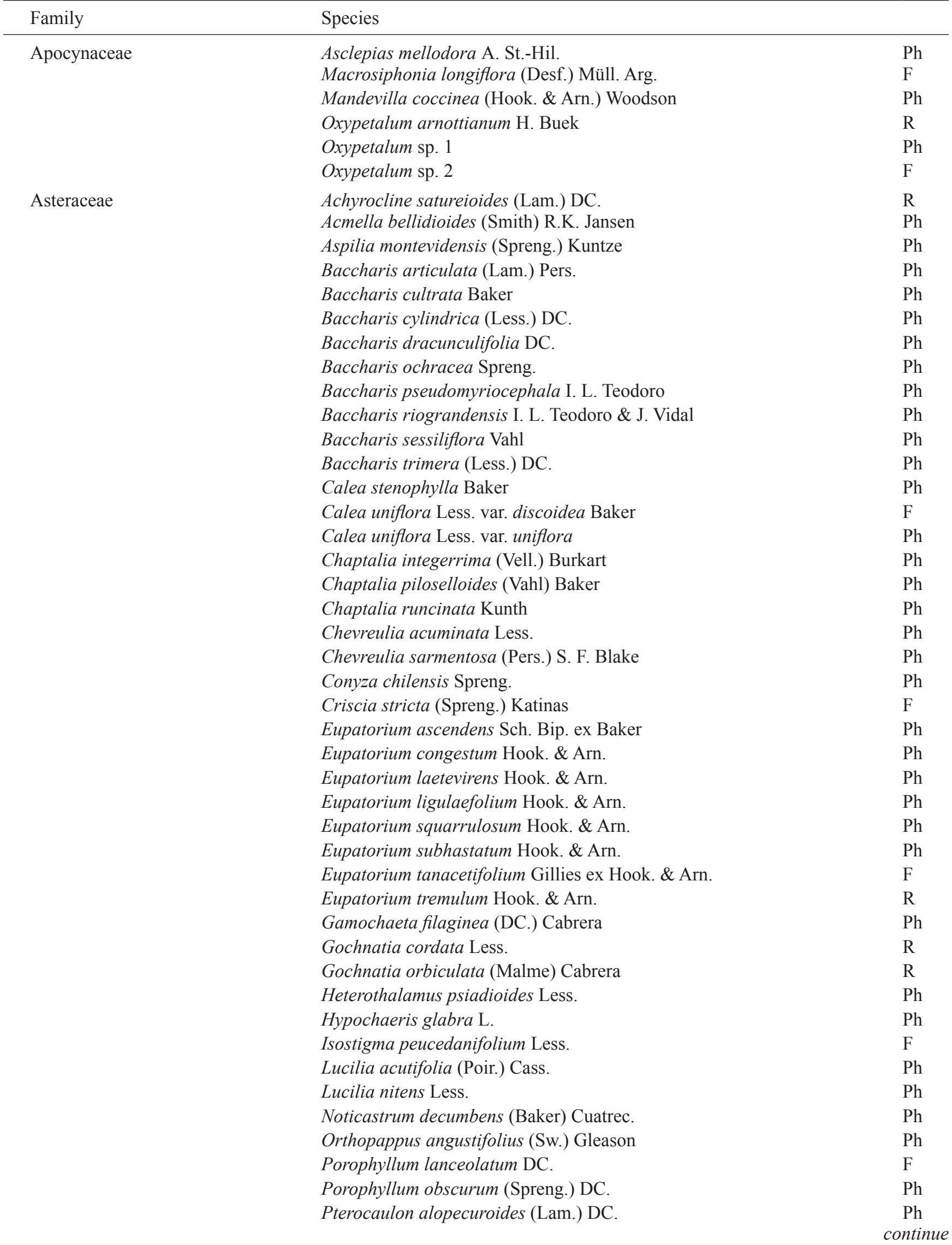


continuation

\begin{tabular}{|c|c|c|}
\hline \multirow[t]{21}{*}{ Family } & \multicolumn{2}{|l|}{ Species } \\
\hline & Pterocaulon angustifolium DC. & $\mathrm{Ph}$ \\
\hline & Pterocaulon polypterum (DC.) Cabrera & $\mathrm{Ph}$ \\
\hline & Schlechtendalia luzulifolia Less. & $\mathrm{F}$ \\
\hline & Senecio cisplatinus Cabrera & $\mathrm{F}$ \\
\hline & Senecio heterotrichius DC. & $\mathrm{F}$ \\
\hline & Senecio leptolobus DC. & $\mathrm{R}$ \\
\hline & Stenachaenium campestre Baker & $\mathrm{Ph}$ \\
\hline & Stenachaenium macrocephalum Griseb. & $\mathrm{R}$ \\
\hline & Stenachaenium megapotamicum Baker & $\mathrm{R}$ \\
\hline & Stenachaenium riedelii Baker & $\mathrm{F}$ \\
\hline & Tagetes minuta $\mathrm{L}$. & $\mathrm{R}$ \\
\hline & Vernonia brevifolia Less. & $\mathrm{Ph}$ \\
\hline & Vernonia flexuosa Sims & $\mathrm{Ph}$ \\
\hline & Vernonia megapotamica Spreng. & $\mathrm{Ph}$ \\
\hline & Vernonia nudiflora Less. & $\mathrm{Ph}$ \\
\hline & Vernonia scorpioides (Lam.) Pers. & $\mathrm{Ph}$ \\
\hline & Vernonia sellowii Less. & $\mathrm{Ph}$ \\
\hline & Vernonia squarrosa (D. Don) Less. & $\mathrm{F}$ \\
\hline & Viguiera immarginata (DC.) Herter & $\mathrm{Ph}$ \\
\hline & Viguiera nudicaulis (Pers.) Baker & $\mathrm{Ph}$ \\
\hline \multirow[t]{2}{*}{ Boraginaceae } & Cordia verbenacea DC. & $\mathrm{F}$ \\
\hline & Moritzia ciliata (Cham.) DC. ex Meisn. & $\mathrm{F}$ \\
\hline \multirow[t]{2}{*}{ Bromeliaceae } & Dyckia choristaminea $\mathrm{Mez}$ & $\mathrm{R}$ \\
\hline & Dyckia leptostachya Baker & $\mathrm{R}$ \\
\hline \multirow[t]{2}{*}{ Cactaceae } & Frailea gracillima (Lem.) Britton \& Rose & $\mathrm{R}$ \\
\hline & Parodia ottonis (Lehm.) N. P. Taylor & $\mathrm{Ph}$ \\
\hline Campanulaceae & Wahlenbergia linarioides (Lam.) A.DC. & $\mathrm{F}$ \\
\hline Cistaceae & Helianthemum brasiliensis (Lam.) Pers. & $\mathrm{Ph}$ \\
\hline Commelinaceae & Commelina erecta $\mathrm{L}$. & $\mathrm{Ph}$ \\
\hline \multirow[t]{5}{*}{ Convolvulaceae } & Convolvulus crenatifolius Ruiz \& Pav. & $\mathrm{F}$ \\
\hline & Dichondra microcalyx (Hallier f.) Fabris & $\mathrm{R}$ \\
\hline & Dichondra sericea $\mathrm{Sw}$. & $\mathrm{Ph}$ \\
\hline & Dichondra sp. & $\mathrm{F}$ \\
\hline & Evolvulus sericeus $\mathrm{Sw}$. & $\mathrm{Ph}$ \\
\hline \multirow[t]{11}{*}{ Cyperaceae } & Bulbostylis capillaris (L.) C. B. Clarke & $\mathrm{Ph}$ \\
\hline & Bulbostylis consanguinea Nees & $\mathrm{Ph}$ \\
\hline & Bulbostylis juncoides (Vahl) Kük. ex Osten & $\mathrm{F}$ \\
\hline & Bulbostylis sphaerocephala (Boeck.) C. B. Clarke & $\mathrm{Ph}$ \\
\hline & Cyperus aggregatus (Willd.) Endl. & $\mathrm{Ph}$ \\
\hline & Cyperus reflexus Vahl var. fraternus (Kunth) Kuntze & $\mathrm{F}$ \\
\hline & Cyperus reflexus Vahl var. reflexus & $\mathrm{F}$ \\
\hline & Rhynchospora gollmeri Boeck. & $\mathrm{F}$ \\
\hline & Rhynchospora rugosa (Vahl) Gale & $\mathrm{Ph}$ \\
\hline & Rhynchospora setigera Griseb. & $\mathrm{Ph}$ \\
\hline & Scleria cf. balansae Maury & $\mathrm{F}$ \\
\hline \multirow[t]{3}{*}{ Euphorbiaceae } & Croton glandulosus L. & $\mathrm{Ph}$ \\
\hline & Croton sp. & $\mathrm{Ph}$ \\
\hline & Euphorbia selloi (Klotzsch \& Garcke) Boiss. & $\mathrm{Ph}$ \\
\hline
\end{tabular}


continuation

\begin{tabular}{|c|c|c|}
\hline Family & Species & \\
\hline \multirow[t]{25}{*}{ Fabaceae } & Aeschynomene falcata (Poir.) DC. & $\mathrm{Ph}$ \\
\hline & Aeschynomene virgata Cav. & $\mathrm{Ph}$ \\
\hline & Centrosema virginianum (L.) Benth. & $\mathrm{Ph}$ \\
\hline & Chamaecrista repens (Vogel) H. S. Irwin \& Barneby & $\mathrm{Ph}$ \\
\hline & Clitoria nana Benth. & $\mathrm{Ph}$ \\
\hline & Collaea stenophylla (Hook. \& Arn.) Benth. & $\mathrm{F}$ \\
\hline & Crotalaria tweediana Benth. & $\mathrm{F}$ \\
\hline & Desmanthus virgatus (L.) Willd. & $\mathrm{Ph}$ \\
\hline & Desmodium incanum DC. & $\mathrm{Ph}$ \\
\hline & Desmodium uncinatum (Jacq.) DC. & $\mathrm{R}$ \\
\hline & Eriosema tacuaremboense Arechav. & $\mathrm{Ph}$ \\
\hline & Galactia marginalis Benth. & $\mathrm{Ph}$ \\
\hline & Indigofera asperifolia Bong. ex Benth. & $\mathrm{F}$ \\
\hline & Lathyrus nervosus Boiss. & $\mathrm{R}$ \\
\hline & Lathyrus subulatus Lam. & $\mathrm{F}$ \\
\hline & Lupinus bracteolaris Desr. & $\mathrm{R}$ \\
\hline & Macroptilium prostratum (Benth.) Urb. & $\mathrm{Ph}$ \\
\hline & Mimosa dolens Vell. & $\mathrm{Ph}$ \\
\hline & Poiretia tetraphylla (Poir.) Burkart & $\mathrm{F}$ \\
\hline & Rhynchosia corylifolia Mart. ex Benth. & $\mathrm{Ph}$ \\
\hline & Rhynchosia diversifolia Micheli & $\mathrm{Ph}$ \\
\hline & Sellocharis paradoxa Taub. & $\mathrm{Ph}$ \\
\hline & Stylosanthes leiocarpa Vogel & $\mathrm{Ph}$ \\
\hline & Stylosanthes montevidensis Vogel & $\mathrm{Ph}$ \\
\hline & Zornia burkartii Vanni & $\mathrm{Ph}$ \\
\hline Gesneriaceae & Sinningia allagophylla (Mart.) Wiehler & $\mathrm{F}$ \\
\hline Hypericaceae & Hypericum caprifoliatum Cham. \& Schltdl. & $\mathrm{R}$ \\
\hline Hypoxidaceae & Hypoxis decumbens L. & $\mathrm{R}$ \\
\hline \multirow[t]{5}{*}{ Iridaceae } & Cypella herbertii (Lindl.) Herb. & $\mathrm{Ph}$ \\
\hline & Herbertia pulchella Sweet & $\mathrm{Ph}$ \\
\hline & Sisyrinchium avenaceum Klatt & $\mathrm{F}$ \\
\hline & Sisyrinchium megapotamicum Malme & $\mathrm{Ph}$ \\
\hline & Sisyrinchium scariosum I.M. Johnst. & $\mathrm{Ph}$ \\
\hline \multirow[t]{3}{*}{ Lamiaceae } & Cunila sp. & $\mathrm{F}$ \\
\hline & Cunila galioides Benth. & $\mathrm{Ph}$ \\
\hline & Glechon ciliata Benth. & $\mathrm{Ph}$ \\
\hline Liliaceae & Clara ophiopogonoides Kunth & $\mathrm{F}$ \\
\hline Linaceae & Cliococca selaginoides (Lam.) C. M. Rogers \& Mildner & $\mathrm{Ph}$ \\
\hline \multirow[t]{3}{*}{ Lythraceae } & Cuphea carthagenensis (Jacq.) J. F. Macbr. & $\mathrm{R}$ \\
\hline & Cuphea glutinosa Cham. \& Schltdl. & $\mathrm{Ph}$ \\
\hline & Cuphea thymoides Cham. \& Schltdl. & $\mathrm{F}$ \\
\hline \multirow[t]{2}{*}{ Malpighiaceae } & Galphimia brasiliensis (L.) A. Juss. & $\mathrm{Ph}$ \\
\hline & Janusia guaranitica (A. St.-Hil.) A. Juss. & $\mathrm{F}$ \\
\hline \multirow[t]{5}{*}{ Malvaceae } & Abutilon malachroides A. St.-Hil. \& Naudin & $\mathrm{F}$ \\
\hline & Krapovickasia macrodon (DC.) Fryxell & $\mathrm{Ph}$ \\
\hline & Krapovickasia urticifolia (A. St.-Hil.) Fryxell & $\mathrm{Ph}$ \\
\hline & Pavonia hastata Cav. & $\mathrm{Ph}$ \\
\hline & Sida rhombifolia L. & $\mathrm{R}$ \\
\hline
\end{tabular}


continuation

\begin{tabular}{|c|c|c|}
\hline \multirow[t]{4}{*}{ Family } & \multicolumn{2}{|l|}{ Species } \\
\hline & Waltheria douradinha A. St.-Hil. & $\mathrm{Ph}$ \\
\hline & Waltheria indica $\mathrm{L}$. & $\mathrm{F}$ \\
\hline & Wissadula glechomifolium (A. St.-Hil.) R.E. Fr. & $\mathrm{Ph}$ \\
\hline \multirow[t]{2}{*}{ Melastomataceae } & Tibouchina gracilis (Bonpl.) Cogn. & $\mathrm{F}$ \\
\hline & Tibouchina sp. & $\mathrm{Ph}$ \\
\hline \multirow[t]{5}{*}{ Myrtaceae } & Campomanesia aurea O. Berg & $\mathrm{Ph}$ \\
\hline & Eugenia dimorpha $\mathrm{O}$. Berg & $\mathrm{R}$ \\
\hline & Psidium formosum (Barb. Rodr.) Burret & $\mathrm{F}$ \\
\hline & Psidium incanum (O. Berg) Burret & $\mathrm{F}$ \\
\hline & Psidium luridum (Spreng.) Burret & $\mathrm{Ph}$ \\
\hline \multirow[t]{2}{*}{ Orchidaceae } & Epidendrum fulgens L. & $\mathrm{F}$ \\
\hline & Skeptrostachys congestiflora (Cogn.) Garay & $\mathrm{Ph}$ \\
\hline Oxalidaceae & Oxalis conorrhiza Jacq. & $\mathrm{F}$ \\
\hline \multirow[t]{3}{*}{ Plantaginaceae } & Plantago myosuros Lam. & $\mathrm{F}$ \\
\hline & Plantago paralias Decne. & $\mathrm{F}$ \\
\hline & Plantago sp. & $\mathrm{Ph}$ \\
\hline \multirow[t]{32}{*}{ Poaceae } & Agenium villosum (Nees) Pilg. & $\mathrm{F}$ \\
\hline & Andropogon lateralis Nees & $\mathrm{Ph}$ \\
\hline & Andropogon selloanus (Hack.) Hack. & $\mathrm{Ph}$ \\
\hline & Aristida circinalis Lindm. & $\mathrm{Ph}$ \\
\hline & Aristida filifolia (Arechav.) Herter & $\mathrm{Ph}$ \\
\hline & Aristida flaccida Trin. \& Rupr. & $\mathrm{Ph}$ \\
\hline & Aristida jubata (Arechav.) Herter & $\mathrm{F}$ \\
\hline & Aristida laevis (Nees) Kunth & $\mathrm{Ph}$ \\
\hline & Aristida spegazzinii Arechav. & $\mathrm{Ph}$ \\
\hline & Axonopus affinis Chase & $\mathrm{R}$ \\
\hline & Axonopus siccus (Nees) Kuhlm. & $\mathrm{Ph}$ \\
\hline & Axonopus suffultus (Mikan ex Trin.) Parodi & $\mathrm{Ph}$ \\
\hline & Briza lamarckiana Nees & $\mathrm{Ph}$ \\
\hline & Briza macrostachya (J. Presl) Steud. & $\mathrm{F}$ \\
\hline & Briza minor L. & $\mathrm{R}$ \\
\hline & Briza subaristata Lam. & $\mathrm{Ph}$ \\
\hline & Briza uniolae (Nees) Nees ex Steud. & $\mathrm{Ph}$ \\
\hline & Bromus auleticus Trin. ex Nees & $\mathrm{F}$ \\
\hline & Bromus catharticus Vahl & $\mathrm{F}$ \\
\hline & Calamagrostis alba (J. Presl) Steud. & $\mathrm{F}$ \\
\hline & Calamagrostis viridiflavescens (Poir.) Steud. & $\mathrm{F}$ \\
\hline & Cortaderia selloana Asch. \& Graebn. & $\mathrm{R}$ \\
\hline & Cynodon dactylon (L.) Pers. & $\mathrm{R}$ \\
\hline & Danthonia cirrata Hack. \& Arechav. & $\mathrm{Ph}$ \\
\hline & Dichanthelium sabulorum (Lam.) Gould \& C. A. Clark & $\mathrm{Ph}$ \\
\hline & Digitaria insularis (L.) Fedde & $\mathrm{F}$ \\
\hline & Eleusine tristachya (Lam.) Lam. & $\mathrm{F}$ \\
\hline & Elyonurus candidus (Trin.) Hack. & $\mathrm{F}$ \\
\hline & Elyonurus sp. & $\mathrm{Ph}$ \\
\hline & Eragrostis lugens Nees & $\mathrm{Ph}$ \\
\hline & Eragrostis neesii Trin. & $\mathrm{F}$ \\
\hline & Eragrostis perennis Döll & $\mathrm{F}$ \\
\hline
\end{tabular}


continuation

\begin{tabular}{|c|c|c|}
\hline \multirow[t]{2}{*}{ Family } & \multicolumn{2}{|l|}{ Species } \\
\hline & Eragrostis plana Nees & $\mathrm{F}$ \\
\hline & Eragrostis polytricha Nees & $\mathrm{Ph}$ \\
\hline & Eragrostis sp. & $\mathrm{Ph}$ \\
\hline & Eustachys distichophylla (Lag.) Nees & $\mathrm{F}$ \\
\hline & Eustachys uliginosa (Hack.) Herter & $\mathrm{F}$ \\
\hline & Leptocoryphium lanatum (Kunth) Nees & $\mathrm{Ph}$ \\
\hline & Melica brasiliana Ard. & $\mathrm{F}$ \\
\hline & Melinis repens (Willd.) Zizka & $\mathrm{F}$ \\
\hline & Panicum bergii Arechav. & $\mathrm{F}$ \\
\hline & Panicum chaseae Roseng., B. R. Arrill. \& Izag. & $\mathrm{Ph}$ \\
\hline & Panicum olyroides Kunth & $\mathrm{F}$ \\
\hline & Paspalum dilatatum Poir. & $\mathrm{F}$ \\
\hline & Paspalum mandiocanum Trin. & $\mathrm{F}$ \\
\hline & Paspalum notatum Flüggé & $\mathrm{F}$ \\
\hline & Paspalum paniculatum $\mathrm{L}$. & $\mathrm{F}$ \\
\hline & Paspalum paucifolium Swallen & $\mathrm{F}$ \\
\hline & Paspalum plicatulum Michx. & $\mathrm{Ph}$ \\
\hline & Paspalum quarinii Morrone \& Zuloaga & $\mathrm{F}$ \\
\hline & Paspalum urvillei Steud. & $\mathrm{F}$ \\
\hline & Phalaris angusta Nees ex Trin. & $\mathrm{F}$ \\
\hline & Piptochaetium montevidense (Spreng.) Parodi & $\mathrm{Ph}$ \\
\hline & Piptochaetium ruprechtianum E. Desv. & $\mathrm{Ph}$ \\
\hline & Piptochaetium stipoides (Trin. \& Rupr.) Hack. & $\mathrm{F}$ \\
\hline & Saccharum cf. asperum (Nees) Steud. & $\mathrm{F}$ \\
\hline & Schizachyrium imberbe (Hack.) A. Camus & $\mathrm{Ph}$ \\
\hline & Schizachyrium microstachyum (Ham.) Roseng., B. R.Arrill. \& Izag. & $\mathrm{Ph}$ \\
\hline & Schizachyrium spicatum (Spreng.) Herter & $\mathrm{Ph}$ \\
\hline & Schizachyrium tenerum Nees & $\mathrm{Ph}$ \\
\hline & Setaria parviflora (Poir.) Kerguélen & $\mathrm{Ph}$ \\
\hline & Setaria vaginata Spreng. & $\mathrm{Ph}$ \\
\hline & Sorghastrum albescens (E. Fourn.) Beetle & $\mathrm{F}$ \\
\hline & Sporobolus camporum Swallen & $\mathrm{Ph}$ \\
\hline & Sporobolus indicus (L.) R. Br. & $\mathrm{R}$ \\
\hline & Steinchisma hians (Elliott) Nash & $\mathrm{F}$ \\
\hline & Stipa cf. filiculmis Del. & $\mathrm{F}$ \\
\hline & Stipa filifolia Nees & $\mathrm{Ph}$ \\
\hline & Stipa megapotamia Spreng. ex Trin. & $\mathrm{F}$ \\
\hline & Stipa melanosperma J. Presl var. melanosperma & $\mathrm{F}$ \\
\hline & Stipa nutans Hack. & $\mathrm{F}$ \\
\hline & Stipa sp. & $\mathrm{Ph}$ \\
\hline & Trachypogon montufarii (Kunth) Nees & $\mathrm{Ph}$ \\
\hline & Urochloa decumbens (Stapf) R. D.Webster & $\mathrm{F}$ \\
\hline Polygalaceae & Monnina cardiocarpa A. St.-Hil. & $\mathrm{Ph}$ \\
\hline & Polygala extraaxillaris Chodat & $\mathrm{Ph}$ \\
\hline Rubiaceae & Diodella apiculata (Willd. ex Roem. \& Schult.) Delprete & $\mathrm{Ph}$ \\
\hline & Galianthe fastigiata Griseb. & $\mathrm{Ph}$ \\
\hline & Galium hirtum Lam. & $\mathrm{Ph}$ \\
\hline & Galium richardianum (Gillies ex Hook. \& Arn.) Endl. ex Walp. & $\mathrm{Ph}$ \\
\hline & Richardia grandiflora (Cham. \& Schltdl.) Steud. & $\mathrm{Ph}$ \\
\hline
\end{tabular}


continuation

\begin{tabular}{|c|c|c|}
\hline Family & Species & \\
\hline \multirow{7}{*}{ Scrophulariaceae } & $\begin{array}{l}\text { Richardia humistrata (Cham. \& Schltdl.) Steud. } \\
\text { Spermacoce canitata Ruiz \& Pav }\end{array}$ & $\begin{array}{l}\mathrm{Ph} \\
\mathrm{Ph}\end{array}$ \\
\hline & Spermacoce verticillata $\mathrm{L}$. & $\mathrm{Ph}$ \\
\hline & Angelonia integerrima Spreng. & $\mathrm{Ph}$ \\
\hline & Buchnera integrifolia Larrañaga & $\mathrm{Ph}$ \\
\hline & Buddleja thyrsoides Lam. & $\mathrm{R}$ \\
\hline & Gerardia communis Cham. \& Schltdl. & $\mathrm{Ph}$ \\
\hline & Scoparia ericacea Cham. \& Schltdl. & $\mathrm{Ph}$ \\
\hline Smilacaceae & Smilax cf. campestris Griseb. & $\mathrm{F}$ \\
\hline \multirow[t]{4}{*}{ Solanaceae } & Calibrachoa ovalifolia (Miers) Stehmann \& Semir & $\mathrm{F}$ \\
\hline & Nicotiana alata Link \& Otto & $\mathrm{F}$ \\
\hline & Petunia integrifolia (Hook.) Schinz \& Thell. & $\mathrm{Ph}$ \\
\hline & Solanum sisymbrifolium Lam. & $\mathrm{F}$ \\
\hline Symplocaceae & Symplocos uniflora (Pohl) Benth. & $\mathrm{F}$ \\
\hline Turneraceae & Piriqueta selloi Urb. & $\mathrm{Ph}$ \\
\hline Urticaceae & Urtica circularis (Hicken) Sorarú & $\mathrm{R}$ \\
\hline \multirow[t]{7}{*}{ Verbenaceae } & Glandularia incisa (Hook.) Tronc. & $\mathrm{F}$ \\
\hline & Glandularia platensis (Spreng.) Schnack \& Covas & $\mathrm{Ph}$ \\
\hline & Glandularia subincana Tronc. & $\mathrm{Ph}$ \\
\hline & Lantana montevidensis (Spreng.) Briq. & $\mathrm{Ph}$ \\
\hline & Stachytarpheta cayennensis (Rich.) Vahl & $\mathrm{F}$ \\
\hline & Verbena lindmani Briq. & $\mathrm{R}$ \\
\hline & Verbena pseudojuncea Gay & $\mathrm{Ph}$ \\
\hline \multirow{2}{*}{ Undetermined } & Dicot. 1 & $\mathrm{Ph}$ \\
\hline & Dicot. 2 & $\mathrm{Ph}$ \\
\hline
\end{tabular}

The structural survey carried out has found 161 species, distributed in 30 families. The most diverse families in the structural survey were Asteraceae (45 species), Poaceae (33 species) and Fabaceae (17 species). However, considering the accumulated IV per family, Poaceae had the highest value (33.21\%), and five of the top-ten IV-ordered species belong to this family (table 2 ). The accumulated IV for Asteraceae and Fabaceae was respectively $28.57 \%$ and $7.22 \%$. The low value for Fabaceae characterized it as a diverse family with low contribution on frequency or cover in the study site. The species with the highest IV were particularly grasses, the prostrate Rubiaceae Richardia grandiflora and some species of Asteraceae (table 2). The diversity and evenness indexes obtained for the community were $\mathrm{H}^{\prime}=4.51$ nats ind ${ }^{-1}$ and $\mathrm{E}=0.86$.

Results from the similarity analysis among Morro do Osso, Morro da Polícia and Morro Santana indicated similar grassland community composition in the three granitic hills analyzed (table 3 ). Although the values themselves are not remarkably high (average of 33\% similarity), they are very close to each other (0.03 standard deviation). There are 57 common species among the three surveys. Many of these species are dominant grasses like Andropogon lateralis, Aristida flaccida and Trachypogon montufarii, suggesting similar community structure and landscape on the different study sites. Moreover, Stipa filifolia, an endemic grass with distribution restricted to Brazilian granitic hills, was also found in the three surveys. Besides grasses, many forb and sub-shrub species of Baccharis, Chaptalia and Eryngium are common among the areas, as well as some rare species like the cactus Parodia ottonis.

Mean species number found per plot $\left(1 \mathrm{~m}^{2}\right)$ was 27.10 (standard deviation $=4.96$ ). Plots with lowest and highest species richness had respectively 15 and 35 species. Mean vegetation height on the sampled area was $28.87 \mathrm{~cm}$ (standard deviation 8.62) and open soil and litter were present in all sampling units, the first with $4.07 \%$ (standard deviation $=13.11$ ) of mean absolute cover per plot unit and the latter with $0.55 \%$ (standard deviation $=0.86$ ). 
Table 2. Grassland species sampled in the structural survey, decreasingly ordered by IV. Only the top-20 IV-valued species are shown. (IV = importance value; $\mathrm{N}=$ number of sampling units in which the species was accounted; $\mathrm{RF}=$ relative frequency; $\mathrm{RC}=$ relative cover).

\begin{tabular}{llcccc}
\hline Family & Species & IV (\%) & N & RF (\%) & RC (\%) \\
\hline Poaceae & Aristida flaccida & 3.22 & 27 & 2.55 & 3.88 \\
Poaceae & Andropogon lateralis & 2.89 & 27 & 2.55 & 3.23 \\
Rubiaceae & Richardia grandiflora & 2.84 & 31 & 2.93 & 2.75 \\
Poaceae & Axonopus suffultus & 2.68 & 26 & 2.46 & 2.91 \\
Asteraceae & Vernonia nudiflora & 2.36 & 26 & 2.46 & 2.26 \\
Asteraceae & Eupatorium laetevirens & 2.25 & 22 & 2.08 & 2.42 \\
Poaceae & Schizachyrium tenerum & 2.10 & 18 & 1.70 & 2.50 \\
Asteraceae & Vernonia flexuosa & 2.10 & 23 & 2.18 & 2.02 \\
Lamiaceae & Glechon ciliata & 2.04 & 21 & 1.99 & 2.10 \\
Poaceae & Panicum chaseae & 2.03 & 19 & 1.80 & 2.26 \\
Poaceae & Paspalum plicatulum & 1.87 & 20 & 1.89 & 1.86 \\
Apiaceae & Eryngium pristis & 1.78 & 12 & 1.14 & 2.42 \\
Asteraceae & Aspilia montevidensis & 1.75 & 20 & 1.89 & 1.62 \\
Fabaceae & Galactia marginalis & 1.75 & 20 & 1.89 & 1.62 \\
Cyperaceae & Rhynchospora rugosa & 1.75 & 20 & 1.89 & 1.62 \\
Asteraceae & Calea uniflora var. uniflora & 1.75 & 19 & 1.80 & 1.70 \\
Convolvulaceae & Evolvulus sericeus & 1.67 & 19 & 1.80 & 1.53 \\
Poaceae & Trachypogon montufarii & 1.63 & 14 & 1.32 & 1.94 \\
Euphorbiaceae & Croton sp. 1 & 1.62 & 18 & 1.70 & 1.53 \\
Poaceae & Axonopus siccus & 1.60 & 15 & 1.42 & 1.78 \\
\hline Total & & 41.70 & - & 39.45 & 43.94 \\
\hline
\end{tabular}

Clustering and ordination analysis - Cluster analysis denoted the existence of two sharp groups among the sampling units (figure 2). Cluster groups coincided with the two major grassland-covered areas that were distinguished by a natural environmental bias caused by fire disturbance due to recent burn on the eastern slope (axis 1 variation; figure 3). The majority of the east plots formed a compact distribution of points on figure 3, whereas the west plots shaped a more scattered pattern (axis 2 variation). Because of the great coincidence among cluster groups and the set of plots located in the same slope, further analyses were done according to plot location.

Species plotted on figure 3 are related to differences in floristic and structural composition between the two

Table 3. Jaccard similarity indexes between our study (Morro do Osso) and two others granitic hills from Porto Alegre region. Data for Morro Santana and Morro da Polícia obtained respectively from Overbeck et al. (2006) and Boldrini et al. (1998). "Sampled Species" values represent taxa identified to the specific level.

\begin{tabular}{lccc}
\hline & Morro do Osso & Morro Santana & Morro da Polícia \\
\hline Morro do Osso & 1 & & \\
Morro Santana & 0.35897 & 1 & 1 \\
Morro da Polícia & 0.33884 & 0.28736 & 171 \\
\hline Sampled species & $152 \mathrm{e}$ & 165 & 71 \\
Exclusive species & 43 & 64 & 2829 points \\
Sampling method & $39 \times 1 \mathrm{~m}^{2}$ quadrats & $48 \times 0.75 \mathrm{~m}^{2}$ quadrats & \\
\hline
\end{tabular}




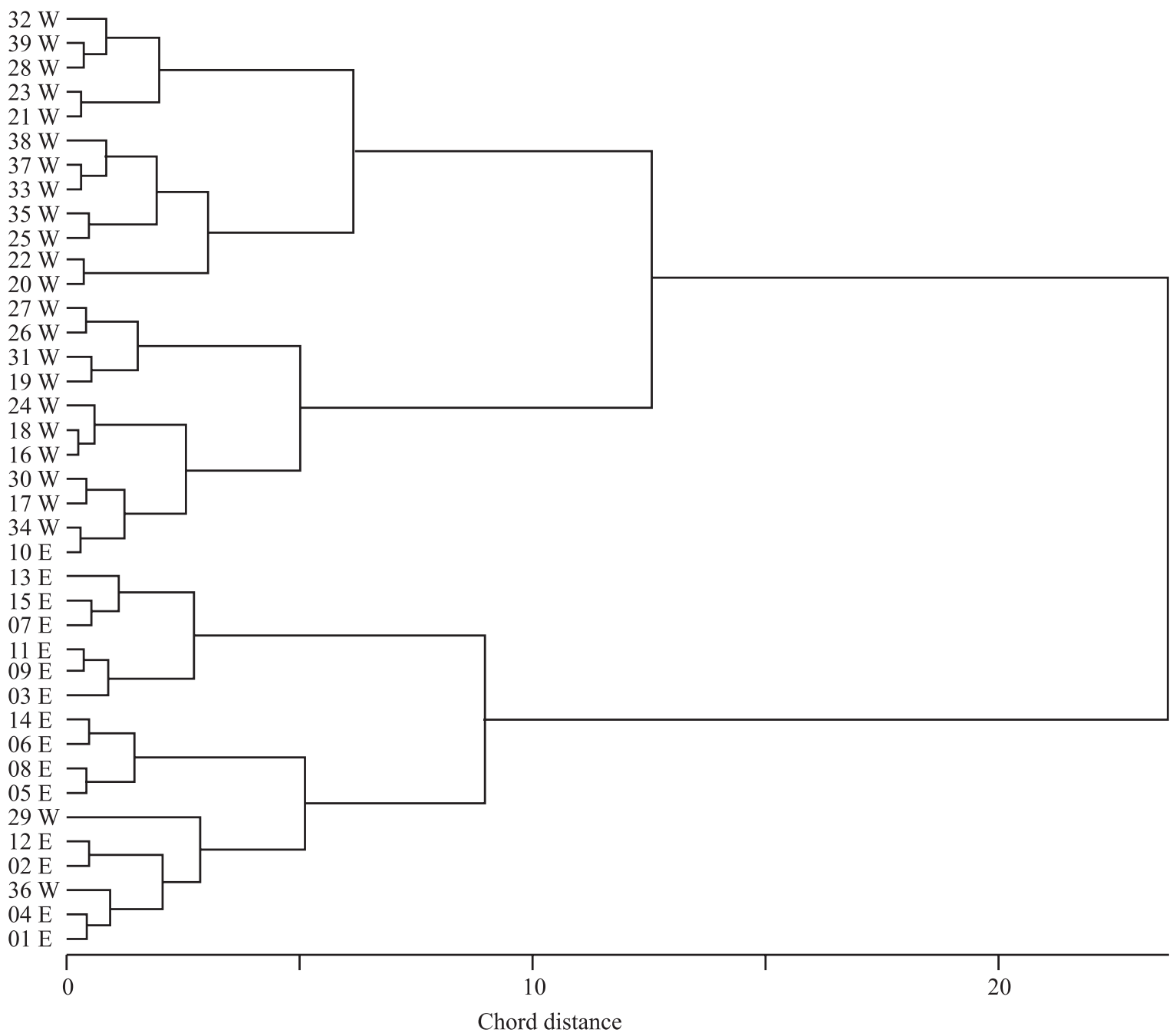

Figure 2. Cluster analysis diagram obtained for the 39 sampling plots described by cover value of species, litter and open soil variables. Only two groups are statistically supported by bootstrap analysis $(P>0.1)$. Incremental sum of squares (horizontal axis) was used as the clustering criterion and chord distance as the dissimilarity measure (E1-E16 = plots in the east grassland area; $\mathrm{W} 17-\mathrm{W} 39=$ plots in the west grassland area).

major grassland areas. These differences can be better observed by species performances (figure 4) in each plot group. East plots presented a total of 111 species, being 32 exclusive of this area, whereas for the west plots 129 species were surveyed in total, being 50 exclusive species. Even with these given floristic peculiarities, there are no statistical differences in richness between the areas (east $=27.75$ species per plot; west $=26.65$ ) .

Grass species such as Schizachyrium tenerum, Trachypogon montufarii, Axonopus siccus and Aristida filifolia had their performance decreased in the east area (compare table 2 and figure 4), whereas Schizachyrium microstachyum, Eragrostis polytricha (grasses), Eryngium horridum, Pterocaulon angustifolium and Vernonia flexuosa (forbs) increased their performance. Andropogon lateralis, Aristida flaccida, Axonopus suffultus (grasses), Vernonia nudiflora (sub-shrub), Eupatorium laetevirens and Aspilia montevidensis (forbs) were dominant species that maintained their relative cover proportion in both areas. The first two grasses, however, had proportionally more cover in east plots (figure 4).

There was no difference considering vegetation height in both grassland areas (east plots: $26.68 \mathrm{~cm}$; west plots: $28.69 \mathrm{~cm}$ ). In addition, open soil mean cover had no statistical difference between the areas, albeit the slight difference between east and west plots (mean of 1.62 and 1.26 , respectively) and the visual difference observed in the field. However, litter presented statistical difference between the areas (mean of 1.06 and 1.39 for east and west plots, respectively; $P=0.03$ ). 


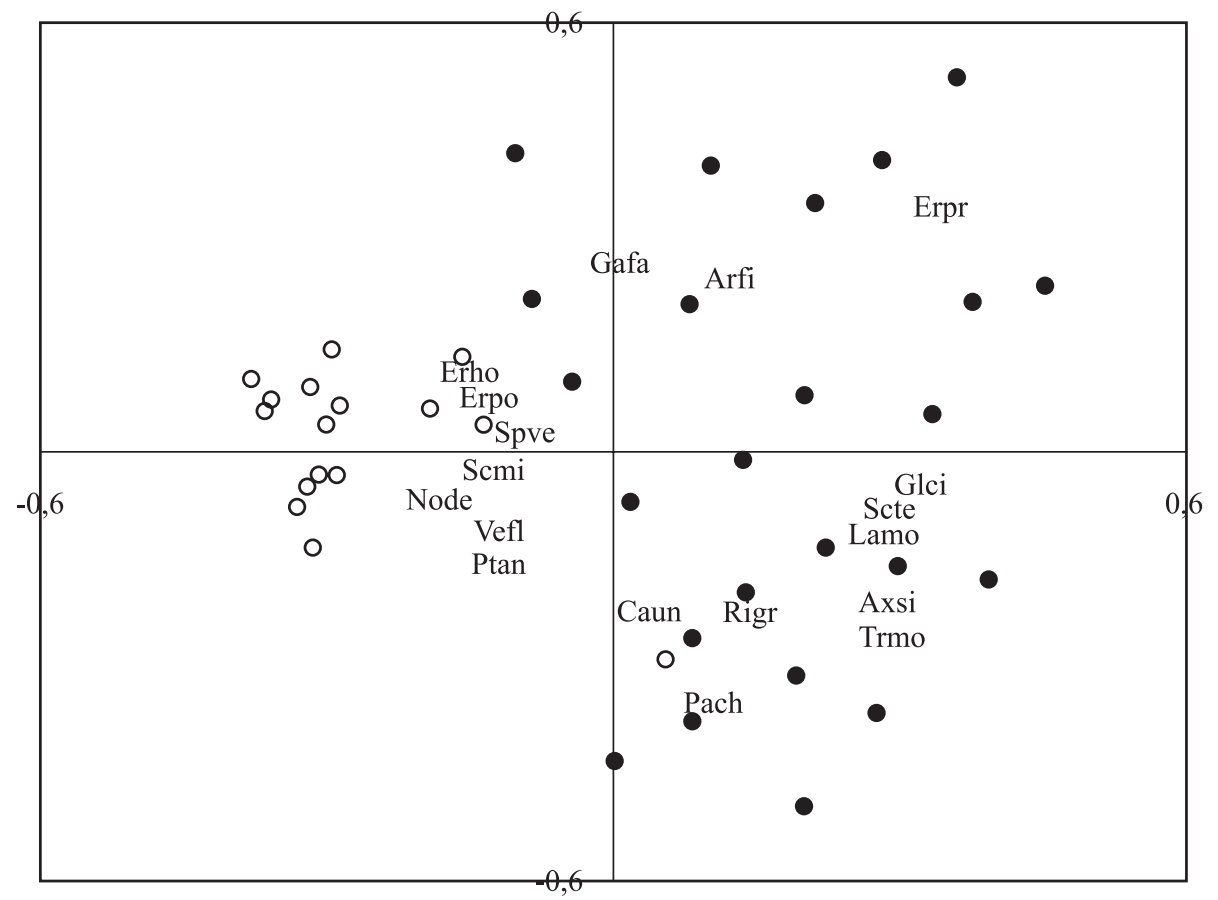

Figure 3. Ordination diagram by PCoA of the 39 sampling plots on the cover value of species, litter and open soil. Plot labels are identified according to main aspect (east; west) of grassland area. Chord distance was used as dissimilarity measure. The ordination axes sum $20 \%$ of total variation. Variables correlated more than 0.45 with the ordination axes were plotted in the diagram $($ Arfi $=$ Aristida filifolia; Axsi $=$ Axonopus siccus; Caun $=$ Calea uniflora; Erpo $=$ Eragrostis polytricha; Erho $=$ Eryngium horridum $;$ Erpr $=$ Eryngium pristis $; \mathrm{Gafa}=$ Galianthe fastigiata $;$ Glci $=$ Glechon ciliata $;$ Lamo $=$ Lantana montevidensis $;$ Node $=$ Noticastrum decumbens $;$ Pach $=$ Panicum chaseae $;$ Ptan = Pterocaulon angustifolium $;$ Rigr $=$ Richardia grandiflora $; \mathrm{Scmi}=$ Schizachyrium microstachyum; $\mathrm{Scte}=$ Schizachyrium tenerum $;$ Spve $=$ Spermacoce verticillata $; \mathrm{Trmo}=$ Trachypogon montufarii; Vefl = Vernonia flexuosa ; empty circles = east; full cicles $=$ west $)$.

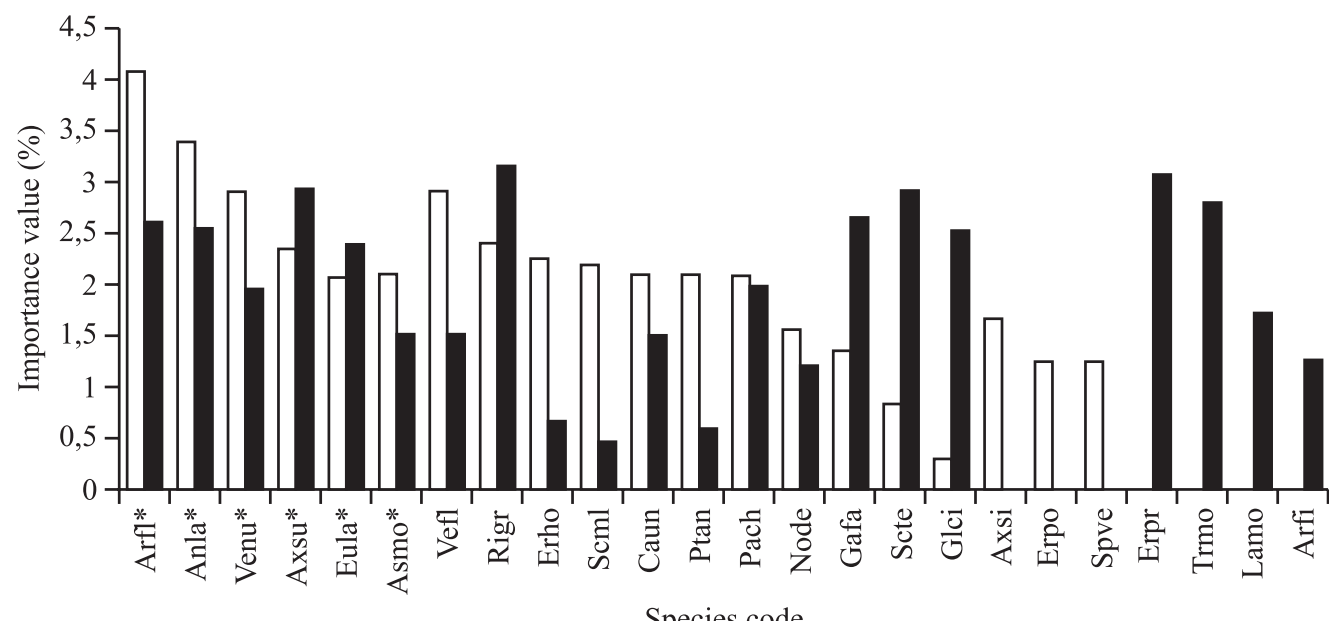

Figure 4. Importance value of typical species of plots representing east (recent burned site) and west grassland areas (species shown in figure 3) and the first five species (*) which are well represented in both areas (Arfl = Aristida flaccida; Anla = Andropogon lateralis; Venu = Vernonia nudiflora $;$ Axsu = Axonopus suffultus; Eula = Eupatorium laetevirens; Asmo = Aspilia montevidensis $;$ Vefl = Vernonia flexuosa $;$ Erho $=$ Eryngium horridum $; \mathrm{Scmi}=$ Schizachyrium microstachyum $;$ Pach $=$ Panicum chaseae $;$ Rigr = Richardia grandiflora $;$ Caun = Calea uniflora $;$ Ptan = Pterocaulon angustifolium; $\mathrm{Gafa}=$ Galianthe fastigiata; $\mathrm{Scte}=$ Schizachyrium tenerum $; \mathrm{Glci}=$ Glechon ciliata $; \mathrm{Axsi}=$ Axonopus siccus $;$ Erpo $=$ Eragrostis polytricha $; \mathrm{Spve}=$ Spermacoce verticillata $;$ Node $=$ Noticastrum decumbens $;$ Erpr $=$ Eryngium pristis $;$ Trmo $=$ Trachypogon montufarii $;$ Lamo $=$ Lantana montevidensis; Arfi = Aristida filifolia $;$ empty bars = east; full bars = west $)$. 


\section{Discussion}

Diversity in grassland formations presented in studies carried out in Rio Grande do Sul State are quite variable, depending on the method and the region surveyed. In a flat region in the middle of the State, different efforts have found less species when compared to the present study. Focht \& Pillar (2003) have found 148 grassland species with vegetation patterns related to relief position and other factors, such as soil moisture, on a study located approximately $35 \mathrm{~km}$ west from ours. On the other hand, granitic grassland formations tend to present high species richness, as showed by Boldrini et al. (1998) in a study based on point method on Morro da Polícia, where 189 species were surveyed. In another similar hill (Morro Santana), 165 species were recorded by plot survey in a grassland area that burns in intervals of three to five years (Overbeck et al. 2006).

The survey accomplished at Morro da Polícia by Boldrini et al. (1998) showed more exclusive species (71) and more overall species (171 fully identified species), probably due to a more exhausting sampling process (18 transects with 2829 points). The survey at Morro Santana (Overbeck et al. 2006) was carried out in 48 plots of 0.75 square meters, a sampling process similar to the one we have implemented at Morro do Osso, and resulted in 165 species (64 exclusive) identified to the specific level. Our survey, comprising the smaller total area among the three hills, showed 43 exclusive species. We consider that, among the exclusive species found in our survey, very few are actually exclusive, and their apparent exclusiveness could be simply related to different collection efforts, as the three compared areas are spatially and floristically close to each other.

Considering a broad study of Porto Alegre's hills region, 522 grassland species were pointed out in a survey on ten granitic hills, not including Morro do Osso (Aguiar et al. 1986). The occurrence of many concomitant species among the lists of these studies and ours indicates a rather continuous flora along the granitic hills. In addition, despite the species richness differences estimated by the similarity indexes for Morro Santana, Morro da Polícia and the present study, there is a very similar species composition among these areas, with an equivalent species-family distribution and many common dominant species. This point may corroborate Rambo's idea that these granitic hills were refuge areas during ocean transgression times (Rambo 1954), so that the extant grassland vegetation configures a remnant of different climate conditions (Behling 2002, Bredenkamp et al. 2002).
Presence of endemic and rare species in the study site is considerably high. Rambo (1954), in a classic paper concerning the southern Brazilian flora, emphasized plant endemism to be outstanding in Porto Alegre area, mostly comprising herbaceous species and genera. Although the endemic species list this author presents have greatly diminished at present due to growing collection effort and subsequent increase in the distribution area of many southern Brazilian species, some species deserve attention concerning their particular occurrence on granitic hills, such as Moritzia ciliata and Stipa filifolia. Other species, such as Dyckia choristaminea, have their distribution area limited to Porto Alegre region, whereas Baccharis riograndensis, Sellocharis paradoxa and Eugenia dimorpha have a broader distribution in the State of Rio Grande do Sul. Besides these, Heterothalamus psiadioides and Dyckia leptostachya have their distribution limited to the southernmost Brazilian state, whereas Eryngium horridum and E. ciliatum have the state as their northern distributional limit, also occurring on northern Uruguay and Argentina

Eleven species presented in the floristic list of this study are categorized as extinction-threatened by official governmental list (Sema 2003). Among them, eight are labeled as vulnerable and three as endangered. None of the endangered species (Dyckia choristaminea, Frailea gracillima and Schlechtendalia luzulifolia) and only four of the vulnerable ones (Gomphrena graminea, Mandevilla coccinea, Parodia ottonis and Waltheria douradinha) were accounted for in the structural survey, suggesting scarcity in the area. The other vulnerable species are Gochnatia cordata, G. orbiculata, Moritzia ciliata and Stenachaenium macrocephalum. Only one individual of $M$. ciliata was sampled, and very few were seen in subsequent expeditions. This evidence of possible population decrease is concerning, as this species is considered, farther than threatened, rare in Brazilian granitic grasslands. The scarcity of $S$. luzulifolia seen on the field is also concerning, as the distribution of this species has Porto Alegre city region as its northern limit (Mondin \& Baptista 1996).

The absolute values of diversity and evenness indexes obtained for the presented community were supposedly higher (4.51 nats ind ${ }^{-1}$ and 0.86 , respectively) than for Morro da Polícia (4.01 nats ind ${ }^{-1}$ and 0.76; Boldrini et al. 1998). Considering that species richness was lower in our area (161 for our study and 189 for Boldrini et al. 1998), the evenness in species performance at Morro do Osso survey should explain the higher Shannon diversity. Another important point is the recent burn undergone in 
part of the sampling area, which may have contributed to the high diversity index obtained for the community. Overbeck et al. (2005, 2006), working in Morro Santana, have shown that fire disturbance clearly leads to a short term increase in species richness and diversity (Denslow 1985, Harrison et al. 2003), but does not significantly affect overall community species composition. The maintenance of composition, alongside the short-term diversity peak and the regularly break of high competitors tussock species dominance, could explain the diversity and evenness values for the surveyed community, since fire in local area is a long term disturbance event. Forb species may be outcompeted by tussock grass dominance (Laterra et al. 2003, Overbeck et al. 2005). This may be first perceived only at above-ground vegetation, since many species can survive underground as bud-bank or reserve organs waiting for better opportunities, but forb submission may occur if disturbance intervals become long enough to suppress those organs (Rodríguez et al. 2003).

Although cluster and ordination analysis showed a distinct pattern in the studied grassland community, patterns among each clustering group were not uniform. East plots showed more similarity among themselves both in cluster and ordination analysis, suggesting a structural and floristic pattern, probably due to the survival of common fire-resilient species (Müller et al. 2007) and similar post-fire species recruitment (Pillar \& Quadros 1997, Uys et al. 2004). On the other hand, west plots do not share a common trait like recent fire and, moreover, are distributed over a larger and more variable environment. Therefore, the pattern drawn by these plots along the second axis on the ordination diagram showed less spatial cohesion, probably illustrating other environmental differences among sampling plots.

Most floristic changes in disturbed areas are a consequence of covering area reduction of dominant species that play a key role on new species recruitment or changes in species performance and consequently in any community diversity modification (Connell 1978, Huston 1979, Olff \& Ritchie 1998). Although the firedriven dominancy reduction that occurred in part of our study area enabled the sprouting of many exclusive species (32) in the east plots, west grassland plots had much more exclusive species (50), possibly due to the removal of fire-vulnerable species in the first and to the higher number of plot units on the western grassland area (23 west plots; 16 east plots). Eryngium pristis, Lantana montevidensis, Trachypogon montufarii and Stipa filifolia are examples of high performance species present exclusively on the west plot units. Overbeck et al. (2005) pointed out that areas with an intermediate time without fire were the most rich of a comparison considering recently (one year) and long time (more than five years) burned places. As it is, the east overall grassland area might reach its species richness peak within few years after this study and our west group plots may be at an intermediate time without influence of fire, presenting more concentration of species per area.

Although fire had no effect on mean vegetation height of sampling units, it did affect floristic and structural composition between the two major areas analyzed. Open soil cover did not differ as well, but litter cover was higher in west plots, clearly as a consequence of burn effect on east grassland area.

Species composition and performance depicted differences between east and west areas. Eryngium horridum, Eragrostis polytricha, Spermacoce verticillata, Noticastrum decumbens, Schizachyrium microstachyum and Pterocaulon angustifolium, which were better represented on our recently burned area, are typically present in early successional stages and altered areas (Eggers \& Porto 1994, Heringer \& Jacques 2002). Quadros \& Pillar (2001), working on a south Brazilian grassland, have also focused on floristic changes after fire and grazing disturbance. They have discussed that vegetation showed greater resilience to fire treatments, albeit remarkably differences were seen in initial observations.

The similarity we have found among the granitic hills, as well as the presence of rare and endemic species among them, denotes a historical continuous flora (at least on grasslands communities) along these formations, corroborating the shared natural history of the area. Besides that, the great diversity observed reflects the ecological importance of this site, as an example of great plant diversity of grassland formations. Nevertheless, further research including more granitic hills and a biogeographical approach are considered to be necessary in order to understand the Porto Alegre's granitic hill chain flora as a whole.

Fire disturbance, with the basic effect of diminishing dominance of some species, thus allowing the rising of others, was partly responsible for the differences in species composition although differences in overall diversity were not recordable. The accidental fire held at the study site showed more effect on performance of species, which is temporary, than on their presence or exclusion of the area. Considering the whole area, this contributes to the high diversity level found for the whole community. 
Acknowledgements - The authors are grateful to the Secretaria do Meio Ambiente of Porto Alegre municipality, especially to MsC. Maria Carmen Sestren-Bastos, for the permission of conducting this study. The first author expresses his gratitude for the scholarship given by Fundação de Amparo à Pesquisa do Estado do Rio Grande do Sul.

\section{References}

AGUIAR, L.W., MARTAU, L., SOARES, Z.F., BUENO, O.L., MARIATH, J.E. \& KLEIN, R.M. 1986. Estudo preliminar da flora e vegetação de morros graníticos da região da Grande Porto Alegre, Rio Grande do Sul, Brasil. Iheringia, Série Botânica 34:3-34.

APWEB 2008. Angiosperm Phylogeny Website. Version 9, June 2008. http://www.mobot.org/MOBOT/research/ APweb/. Stevens, P.F. (2001 onwards). (acesso em 08/10/2009)

BEHLING, H. 2002. South and southeast Brazilian grassland during Late Quaternary times: a synthesis. Palaegeography, Palaeclimatology, Palaeoecology 177:19-27.

BEHLING, H., PILLAR, V.D., ORLÓCI, L. \& BAUERMANN, S.G. 2004. Late Quaternary Araucaria forest, grassland (Campos), fire and climate dynamics, studied by high-resolution pollen, charcoal and multivariate analysis of the Cambará do Sul core in southern Brazil. Palaegeography, Palaeclimatology, Palaeoecology 203:277-297.

BEHLING, H., PILlAR, V.D., MÜllER, S.C. \& OVERBECK, G.E. 2007. Late-Holocene fire history in a forest-grassland mosaic in southern Brasil: implications for conservation. Applied Vegetation Science 10:81-90.

BOLDRINI, I.B., MIOTTO, S.T.S., LONGHI-WAGNER, H.M., PILLAR, V.D. \& MARZALL, K. 1998. Aspectos florísticos e ecológicos da vegetação campestre do Morro da Polícia, Porto Alegre, RS, Brasil. Acta Botanica Brasilica 12:89-100.

BRACK, P., RODRIGUES, R.S. \& LEITE, S.L.C. 1998. Morro do Osso: um santuário cercado pela cidade. In Atlas ambiental de Porto Alegre (R. Menegat, M.L. Porto, C.C. Carraro \& L.A.D. Fernandes, eds.). Universidade Federal do Rio Grande do Sul, Porto Alegre, p.80.

BRACK, P., VASQUES, C.L., MARTINS, R.P. \& RODRIGUES, R.S. 2001. Flora. In Flora e Fauna do Parque Natural do Morro do Osso (S.R. Mirapalhete, ed.). SMAM - Secretaria Municipal do Meio Ambiente, Porto Alegre, p.23-45.

BREDENKAMP, G.J., SPADA, F. \& KAZMIERCZAK, E. 2002. On the origin of northern and southern hemisphere grasslands. Plant Ecology 16:209-229.

CONNELL, J.H. 1978. Diversity in tropical rain forests and coral reefs. Science 199:1302-1310.
DENSLOW, J.S. 1985. Disturbance-mediated coexistence of species. In The ecology of natural disturbance and patch dynamics (S.T.A. Pickett \& P.S. White, eds.). Academic Press, San Diego.

EGGERS, L. \& PORTO, M.L. 1994. Ação do fogo em uma comunidade campestre secundária, analisada em bases fitossociológicas. Boletim do Instituto de Biociências da Universidade Federal do Rio Grande do Sul 53:1-88.

FOCHT, T. \& PILLAR, V.D. 2003. Spatial patterns and relations with the site factors in a campos grassland under grazing. Brazilian Journal of Biology 63:423-436.

HARRISON, S., INOUYE, B.D. \& SAFFORD, H.D. 2003. Ecological heterogeneity in the effects of grazing and fire on grassland diversity. Conservation Biology 17:837-845.

HERINGER, I. \& JACQUES, A.V.A. 2002. Composição florística de uma pastagem natural submetida a queima e manejos alternativos. Ciência Rural 32:315-321.

HUSTON, M. 1979. A general hypothesis of species diversity. American Naturalist 113:81-101.

LATERRA, P., VIGNOLIO, O.R., LINARES, M.P., GIAQUINTA, A. \& MACEIRA, N. 2003. Cumulative effects of fire on a tussock pampa grassland. Journal of Vegetation Science 14:43-54.

MAGURRAN, A.E. 1988. Ecological diversity and its measurement. Croom Helm, London.

MANLY, B.F.J. 1997. Randomization, bootstrap and Monte Carlo methods in biology. Chapman \& Hall, London.

MENEGAT, R., HASENACK, H. \& CARRARO, C.C. 1998. As formas da superfície: síntese do Rio Grande do Sul. In Atlas ambiental de Porto Alegre (R. Menegat, M.L. Porto, C.C. Carraro \& L.A.D. Fernandes, eds.). Ed. Universidade Federal do Rio Grande do Sul - UFRGS, Porto Alegre, p.25-34.

MONDIN, C.A. \& BAPTISTA, L.R.M. 1996. Relações biogeográficas da tribo Mutisieae Cass. (Asteraceae), sensu Cabrera, no Rio Grande do Sul. Comunicações do Museu de Ciência e Tecnologia - PUCRS. Série Botânica 2:49-152.

MUELLER-DOMBOIS, D. \& ELLENBERG, H. 1974. Aims and methods of vegetation ecology. John Wiley, New York.

MÜlLER, S.C., OVERBECK, G.E., PFADENHAUER, J. \& PILLAR, V.D. 2007. Plant functional types of woody species related to fire disturbance in forest-grassland ecotones. Plant Ecology 189:1-14.

NIMER, E. 1990. Clima. In Geografia do Brasil: Região Sul (IBGE, ed.). IBGE, Rio de Janeiro, p.151-187.

OLFF, H. \& RITCHIE, M.E. 1998. Effects of herbivores on grassland plant diversity. Trends in Ecology \& Evolution 13:261-265.

OVERBECK, G.E., MÜllER, S.C., PILlAR, V.D. \& PFADENHAUER, J. 2005. Fine-scale post-fire dynamics in southern Brazilian subtropical grassland. Journal of Vegetation Science 16:655-664. 
OVERBECK, G.E., MÜLlER, S.C., PFADENHAUER, J. \& PILLAR, V.D. 2006. Floristic composition, environmental variation and species distribution patterns in a burned grassland in southern Brazil. Brazilian Journal of Biology 66:1073-1090.

OVERBECK, G.E., MÜLLER, S.C., FIDELIS, A., PFADENHAUER, J., PILLAR, V.D., BLANCO, C.C., BOLDRINI, I.I., BOTH, R. \& FORNECK, E.D. 2007. Brazil's neglected biome: the South Brazilian Campos. Perspectives in Plant Ecology, Evolution and Systematics 9:101-116.

PIELOU, E.C. 1969. An introduction to mathematical ecology. Wiley-Interscience, New York.

PILLAR, V.D. 1999a. How sharp are the classifications? Ecology 80:2508-2516.

PILLAR, V.D. 1999b. The bootstrap ordination re-examined. Journal of Vegetation Science 10:895-905.

PILLAR, V.D. 2006. MULTIV sofware para análise multivariada, testes de aleatorização e autoreamostragem "bootstrap", v.2.4.2. Departamento de Ecologia, Universidade Federal do Rio Grande do Sul, Porto Alegre.

PILLAR, V.D. \& ORLÓCI, L. 1993. Character-based community analysis; the theory and an application program. SPB Academic Publishing, The Hague.

PILLAR, V.D. \& ORLÓCI, L. 1996. On randomization testing in vegetation science: multifactor comparisons of relevé groups. Journal of Vegetation Science 7:582-592.
PILLAR, V.D. \& QUADROS, F.L.F. 1997. Grassland-forest boundaries in southern Brazil. Coenoses 12:119-126.

PODANI, J. 2000. Introduction to the exploration of multivariate biological data. Backhuys Publishers, Leiden.

QUADROS, F.L.F. \& PILLAR, V.D. 2001. Dinâmica vegetacional em pastagem natural submetida a tratamentos de queima e pastejo. Ciência Rural 31: 863-868.

RAMBO, B. 1954. Análise histórica da flora de Porto Alegre. Sellowia 6:9-111.

RAMBO, B. 1956. A fisionomia do Rio Grande do Sul. Selbach, Porto Alegre.

RODRÍGUEZ, C., LEONI, E., LEZAMA, F. \& ALTESOR, A. 2003. Temporal trends in species composition and plant traits in natural grasslands of Uruguay. Journal of Vegetation Science 14:433-440.

SEMA 2003. Diário Oficial. Decreto 42099-01/01/2003. http://www.sema.rs.gov.br(Secretaria do Meio Ambiente do Estado do Rio Grande do Sul), Porto Alegre. (acesso em 08/10/2009).

SESTREN-BASTOS, M.C. (ed.) 2006. Plano de manejo participativo do Parque Natural Morro do Osso. SMAM (Secretaria Municipal do Meio Ambiente), Porto Alegre.

UYS, R.G., BOND, W.J. \& EVERSON, T.M. 2004. The effect of different fire regimes on plant diversity southern African grasslands. Biological Conservation 118:489-499. 